\title{
Fault Feature Extraction of Diesel Engine Based on Bispectrum Image Fractal Dimension
}

\author{
Jian Zhang, Chang-Wen Liu, Feng-Rong Bi ${ }^{*}$, Xiao-Bo Bi and Xiao Yang
}

\begin{abstract}
Fault feature extraction has a positive effect on accurate diagnosis of diesel engine. Currently, studies of fault feature extraction have focused on the time domain or the frequency domain of signals. However, early fault signals are mostly weak energy signals, and time domain or frequency domain features will be overwhelmed by strong background noise. In order consistent features to be extracted that accurately represent the state of the engine, bispectrum estimation is used to analyze the nonlinearity, non-Gaussianity and quadratic phase coupling (QPC) information of the engine vibration signals under different conditions. Digital image processing and fractal theory is used to extract the fractal features of the bispectrum pictures. The outcomes demonstrate that the diesel engine vibration signal bispectrum under different working conditions shows an obvious differences and the most complicated bispectrum is in the normal state. The fractal dimension of various invalid signs is novel and diverse fractal parameters were utilized to separate and characterize them. The value of the fractal dimension is consistent with the non-Gaussian intensity of the signal, so it can be used as an eigenvalue of fault diagnosis, and also be used as a non-Gaussian signal strength indicator. Consequently, a symptomatic approach in view of the hypothetical outcome is inferred and checked by the examination of vibration signals from the diesel motor. The proposed research provides the basis for on-line monitoring and diagnosis of valve train faults.
\end{abstract}

Keywords: Engine fault diagnosis, Bispectrum image processing, Fractal, Signal processing

\section{Introduction}

Vibration signals are widely utilized for health condition assessment and fault diagnosis in diesel engine and frequently transfer active data from mechanical elements. Many advanced techniques have been employed to detect and extract features from vibration signals [1-6]. Some conventional fault diagnosis techniques based on vibration signals extract the characteristic quantities from the time domain and frequency domain, statistical indexes, such as peak amplitude, root mean square amplitude, kurtosis and frequency components [7]. Obviously, these indexes simplify the description of the machine condition, but the selection of index directly affects the pattern recognition. Moreover, most frequency spectrums have similar characteristics, which make misjudgements in detecting machine faults [8]. Fourier transforms (FT) and fast Fourier transform (FFT) analysis can be utilized

\footnotetext{
*Correspondence: fr_bi@tju.edu.cn
}

State Key Laboratory of Engines, Tianjin University, Tianjin 300072, China to examine the signal in the regularity area. The application premise of discrete Fourier is that it is supposed that the signal is both stationary and periodic [9]. However, the diesel engine is a composite system in which the combined shock is excited by a complicated mechanism motion and combustion. The vibration signal of the diesel engine is not linear and stationary, under steady operation. Intervallic behavior may or may not occur and the presumption of stationarity may not hold when the flag is recorded for long spans or the fundamental instruments of flag era change. In this manner, the blame vibration highlights of diesel motor are not completely be reflected by time-area waveform and spectral examination.

As of late, some new strategies for blame flag determination with non-stationary signs have been proposed, such as wavelet transform (WT), wavelet packet transform (WPT), ensemble empirical mode decomposition (EEMD) and higher order spectral (HOS) analysis [10-12]. Of these new techniques, WT has been proved to be more appropriate than FT for the vibration signal
Springer Open

(c) The Author(s) 2018. This article is distributed under the terms of the Creative Commons Attribution 4.0 International License (http://creativecommons.org/licenses/by/4.0/), which permits unrestricted use, distribution, and reproduction in any medium, provided you give appropriate credit to the original author(s) and the source, provide a link to the Creative Commons license, and indicate if changes were made. 
examination due to its high time-frequency resolution. WPT method that evolved from the wavelet can divide the signal into an entire frequency band. However, both method is unable to excerpt non-linear connections within the signal or time sequence and will lose phase information between frequency components. Moreover, both methods is subject to the selection of the wavelet basis function. Once the wavelet basis is set, decomposition and reconstruction will no longer change and will no longer be adaptive to signal analysis [13]. EEMD investigation has specific benefits for describing signals at instantaneous frequency. An intelligent diagnosis method based on better EEMD and SVM with a small number of training sets was proposed by Zhang et al. [14] to solve the problems of the poor decomposition accuracy for the short signal. Based on EMD, Shi et al. [15] presented a novel procedure to improve the precision of signal decomposition. EEMD is a powerful tool for nonstationary signal analysis, but this method is frequently confined to experiments and applied research. Moreover, low computational efficiency limits its application for online detection. HOS allows characterization of signals at various confinement levels in time and capacity in signal handling, design acknowledgment, seismology, and machine fault analysis.

HOS examination can be utilized as an intense device for the non-straight dynamical investigation of the engine vibration signals. Firstly, this theoretical approach can suppress Gaussian noise. If a non-Gaussian signal contains Gaussian noise, the noise will be eliminated by calculation of the HOS. Secondly, HOS preserves phase information. Thirdly, HOS can assume a key part in identifying and portraying the sort of non-linearity in a framework from its signals [16]. HOS hypothesis would be a superior approach than conventional time-area and recurrence space strategies for analysis of the engine vibration signals, particularly for the weak and noisy signals. The appliance of HOS for health state assessment and fault judgement of diesel engine has not been widespread. Gu et al. [17] made some excellent work for motor fault diagnosis by analysis of the HOS features of electrical motor current. Other researchers used theory and experiments to analyze gear fault diagnosis using analysis of the bispectrum of vibration signals [18]. Feng et al. [19] used bispectrum analysis technique in fault trait mining of the diesel engine piston-pin. He considered the bispectral feature frequency face as the feature parameter and then used a neural network for pattern recognition. However, the feature frequency face may lose fault information.

Bispectrum is the most extensively used approach and the lowest order of all HOS analysis methods. The bispectrum plan provides supplementary data of the relationships among the dissimilar frequency mechanisms. These plans can be used to distinguish diverse conditions of engine, and the features derived from these plans will guide the identification of different kinds of signals. Extracting fit features from the bispectrum plan to provide decision support for fault diagnoses is an important and difficult research topic. The bispectral characteristic plane in frequency field was used to characterize the data [20]. By bispectral analysis of the vibration data and searching the field of bispectral modulus, characteristic planes in data were determined. The fault characteristics of crankshaft bearing can be extracted efficiently. Bispectrum estimation based on a AR model which is not Gaussian was used to describe the nonlinear and non-Gaussian features of the cylinder vibration response signal [21]. The diesel engine valve train fault type was identified by extracting the maximum peak frequency and amplitude from the main cross slice of the bispectrum. However, the main diagonal slice and the characteristic frequency surface contain part of the bispectrum information, limiting the practical effect of the fault features.

The color spatial distribution is considered to be the texture features of the 2D bispectrum plot and reflects the distribution and intensity of the additional information in the dual frequency domain, and exhibits its self-similarity characteristic. Therefore, it can be characterized by the fractal dimension (FD). FD is broadly utilized as a part of numerous ranges of science, permitting quantitative estimation for fractal qualities of nonlinear frameworks and space-filling limit estimation for signals. Numerous techniques are utilized to evaluate FD [22]. In this investigation, FD of the bispectrum was assessed by the crate checking strategy.

Whatever remains of this paper is organized as takes after. Section 2 presents the essential hypothesis of the bispectrum, picture preparing, and fractal measurement. In Section 3, the blame recreation framework and information procurement framework are displayed. The exploratory outcomes and investigation are appeared in Section 4. At long last, the conclusions are given.

\section{Bispectrum Calculation}

\subsection{Definition of Bispectrum}

HOS is a spectral representation of greater order cumulants of an arbitrary process. For a cyclostationary, discrete stochastic process $x(n)$, the $n$ th-order cumulants of $x(n)$ is denoted by $c_{n, x}\left(m_{1}, m_{2}, \ldots, m_{n-1}\right)$. First-order cumulants of a stationary process is known to be the average:

$$
c_{1, x}(0)=E(x(n)),
$$


where $E(\bullet)$ is mathematical expectation. The second and third-order cumulants of a motionless procedure are demarcated by Eq. (1) as follows:

$$
\begin{aligned}
& c_{2, x}(m)=E(x(n) x(n+m)), \\
& c_{3, x}\left(m_{1}, m_{2}\right)=E\left(x(n) x\left(n+m_{1}\right) x\left(n+m_{2}\right)\right) .
\end{aligned}
$$

HOS are demarcated as FT of the equivalent higherorder cumulants arrays. Actually, the Fourier transform of a second-order cumulant is the conventional power spectrum:

$$
S_{2, x}(\omega)=\sum_{m_{1}=-\infty}^{\infty} c_{2, x}(m) \exp (-j \omega m) .
$$

The third-order spectrum is the FT of the third-order cumulants, which is also called the bispectrum [23]:

$$
\begin{aligned}
S_{3, x}\left(\omega_{1}, \omega_{2}\right)= & \sum_{m_{1}=-\infty}^{\infty} \sum_{m_{2}=-\infty}^{\infty} c_{3, x}\left(m_{1}, m_{2}\right) \\
& \times \exp \left(-j\left(\omega_{1} m_{1}+\omega_{2} m_{2}\right)\right) .
\end{aligned}
$$

Note that in the expressions above, these spectra are given by products of the deterministic time-domain signals Fourier transforms. The bispectrum is a representation of the correlation of the spectrum values and two frequency component, reflecting the skewness of signal features. For linear systems, the bispectrum amplitude is zero when all three are independent. For a nonlinear system, there will be strong correlation at some frequencies. In this way, it can distinguish stage coupling between two frequencies which show up as a third recurrence as the total or distinction of the initial two with a stage that is likewise the aggregate or contrast of the initial two [24]. This marvel is called quadratic stage coupling (QPC) [25].

Generally, a power range is utilized to break down the signs into a progression of recurrence segments. In any case, a power range can't decide if crests at agreeably related positions are stage coupled or not on account of the power range utilizes just the size of the Fourier segments and stage data is ignored. HOS, as bispectrum, are equipped for recognizing stage coupling by utilizing stage data. In this manner the bispectrum can give extra recurrence data that the established power range can't give.

The upsides of utilizing HOS examination can be condensed as takes after [25]:

(1) HOS can be used to suppress the influence of the additive colored noise.

(2) HOS can identify the cause and effect, non-minimum phase system or reconstruction non-minimum phase signal.
(3) HOS can provide additional information due to the Gaussian deviation.

(4) HOS can be used to detect and characterize the nonlinearity of signals and identify the nonlinear systems.

(5) Additional QPC information is available.

\subsection{Calculation of Bispectrum}

There exist a few methods for bispectrum approximation, including models with our without parameters. Each model includes direct and indirect methods. Although the parametric model can provide higher resolution and more phase information using less signal data samples, there may be loss in important feature information, and the method cannot reflect the characteristics of the fault signal. Nonparametric bispectrum estimation usually uses more data samples, but this approach can reduce the estimation variance and improve accuracy. In the present work, the straight method of nonparametric model approximation was accepted and the calculation algorithm process is as follows.

(1) Let $x=\left(x_{1}, x_{2}, \ldots, x_{n}\right)$ be the zero mean observation samples and $f_{s}$ is the sampling frequency. In the domain of the bispectrum, the sampling value of the frequency number is $N_{0}$ and the segment of frequency sampling is $\Delta_{0}=f_{\mathrm{s}} / N_{0}$. Next, segment the data into $k$ possibly overlapping records, where each subgroup contains $M$ notation samples.

(2) Remove the average of each subgroup.

(3) Perform the Fourier transformation for each set of data:

$x_{i}(\lambda)=\frac{1}{M} \sum_{n=1}^{M} x_{i}(n) \cdot \exp (-j 2 \pi n \lambda / M)$,

where $\lambda=0,1, \ldots, M / 2, i=1,2, \ldots, K$.

(4) Calculate the bispectrum of each record founded on the numbers gained from DFT:

$$
\begin{gathered}
\hat{b}_{i}\left(\lambda_{1}, \lambda_{2}\right)=\sum_{p_{1}=-L_{1}}^{L_{1}} \sum_{p_{2}=-L_{1}}^{L_{1}} x_{i}\left(\lambda_{1}+p_{1}\right) \cdot x_{i}\left(\lambda_{2}+p_{2}\right) \\
\cdot x_{i}\left(-\lambda_{1}-\lambda_{2}-p_{1}-p_{2}\right) .
\end{gathered}
$$

(5) At last, find the mean and smooth the bispectrum among the values.

$\hat{B}_{x}\left(\omega_{1}, \omega_{2}\right)=\frac{1}{K} \sum_{i=1}^{K} \hat{b}_{i}\left(\omega_{1}, \omega_{2}\right)$,

where $\omega_{1}=2 \pi f_{\mathrm{s}} \lambda_{1} / N_{0}, \omega_{2}=2 \pi f_{s} \lambda_{2} / N_{0}$. 


\subsection{Bispectrum Image Processing}

The picture processing handling was created utilizing Matlab. The schematic portrayal of the picture handling technique is delineated in Figure 1.

As appeared in Figure 1, the transformation of RGB pictures into grayscale pictures and the ensuing background amendment ought to be done first. Next, the grayscale pictures were improved by histogram evening out and the commotion was evacuated by low-pass filtering. In this progression, work histeq and $\mathrm{fft} 2$ gave by Matlab picture handling tool compartment were connected; the low-pass filter was modified freely. A versatile limit determination named OSTU method [26, 27], which can break down picture histograms consequently and acquire the best edge esteem, was utilized to get paired pictures after the computerized pictures were upgraded. This technique was performed by calling capacity graythresh from the Matlab picture handling tool stash. Next, double pictures were furthermore prepared by picture disintegration, calling capacity strel and imerode. At long last, with the capacity edge, shapes of the pictures were gotten by Candy administrator.

\subsection{Fractal Dimension Calculation}

In fractal geometry, the FD is a measurable amount to demonstrate how totally a fractal seems to fill space [28]. FD is a compelling parameter to evaluate fractal attributes.

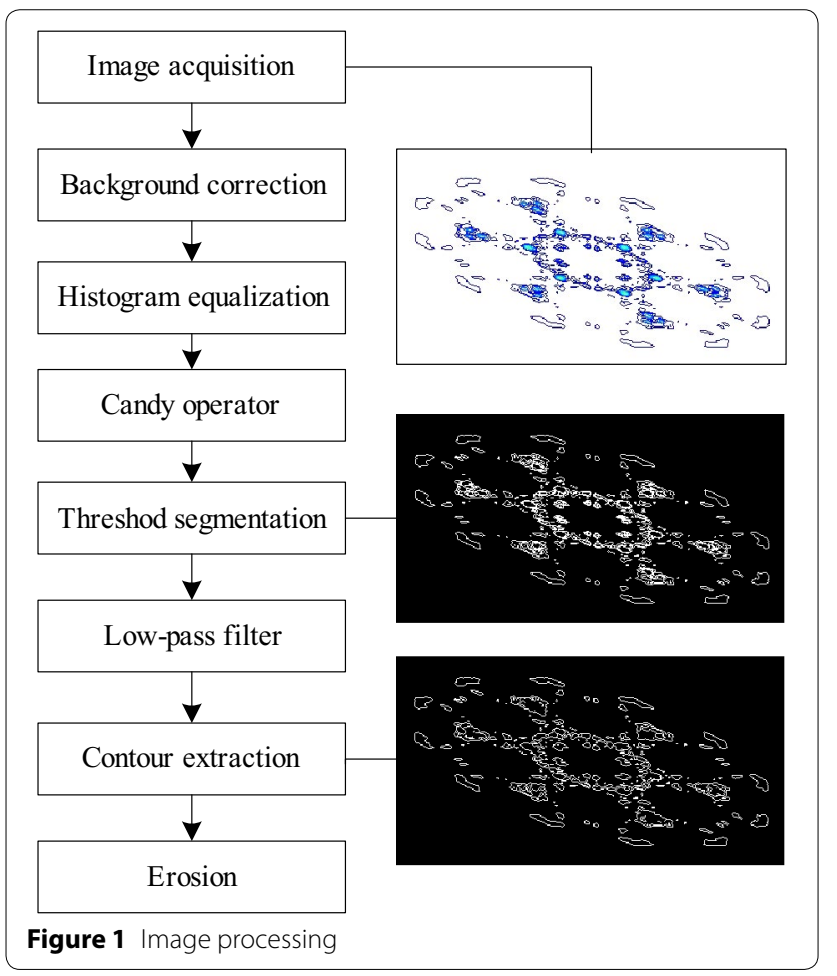

In this paper, $\mathrm{FD}$ of the bispectrum were approximated by the container checking technique as indicated by Theiler [29] with some modification. All the more decisively, each electronic picture was overlaid by a progression of matrices of square boxes of the span of 1-1024 pixels. For a progression of boxes of side length s pixels, the quantity of boxes meeting the shapes of picture by the set $(N)$ was tallied. Fractal structures comply with the power law connection over a scope of length scales, such that [30]:

$$
N(s)=c s^{-D_{B}},
$$

where $D_{B}$ is the case checking fractal measurement, $N(s)$ is the aggregate number of boxes of side length $s$ that cross forms of the bispectrum picture, and $c$ is a consistent. $D_{B}$ is evaluated as the negative slope of a relapse line through the direct piece of the plot of $\lg N(s)$ against $\lg s$, for a succession of scales $s$ :

$$
\lg N(s)=\lg c+\left(-D_{B}\right) \lg s .
$$

\section{Test Setup and Data Acquisition}

In this paper, an inline 6-cylinder diesel engine was tested. The engine works under $1600 \mathrm{r} / \mathrm{min}$ (40\% loads). As shown in Figure 2, the experimental test fix comprises of the six-barrel in-line turbo-charged diesel motor, dynamometer, LMS SCADA III multi-analyzer framework (piezoelectric accelerometer vibration sensors and an information securing framework with $25.6 \mathrm{kHz}$ examining recurrence) and a PC.

The experiment comprises engine valve clearance normal and fault conditions. At the intake and exhaust valve of the first cylinder, vibration signals were collected under preset working fault. The acceleration transducer was installed between the intake valve and exhaust valve. Table 1 shows the fault parameter of the conditions. After the experimental work, the fault and normal vibration signals were recorded by 9 accelerometer sensors using a data acquisition system. The positions of the trembling sensors are shown in Figure 3. The sample time was $30 \mathrm{~s}$. 10 fragments were cut from the collected signals; each fragment lasts $1 \mathrm{~s}$ : 2-3 s, 5-6 s, 7-8 s, 10-11 s, 13-14 s, 16-17 s, 19-20 s, 22-23 s, 24-25 s, 27-28 s. Each fragment contains 26.67 working cycle.

\section{Fault Feature Extraction}

The change of valve clearance can result changes of engine mechanism or the gas exchange process. Changing the engine mechanism will transform the whole character of the engine system. In this case, with the change of valve chain's dynamic character, driving force such as valve-seating impact and impact between valve stems and rocker will also be different. Thus the vibration 


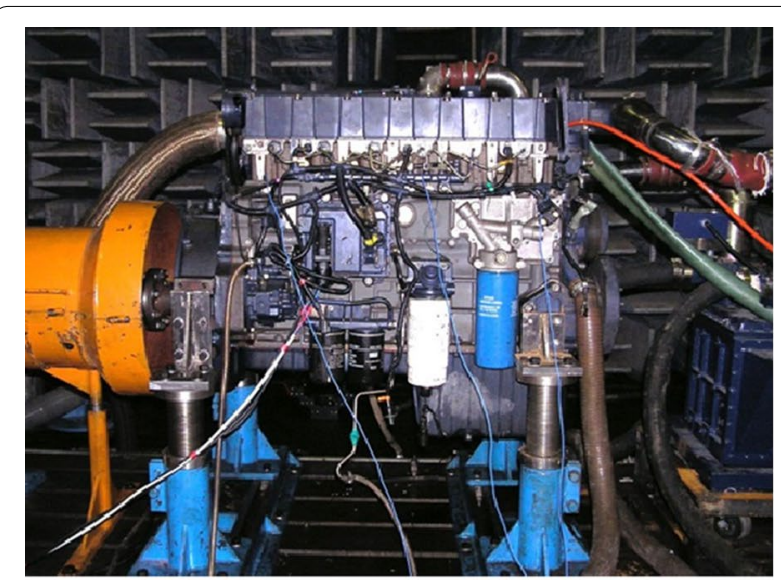

(a) Diesel engine

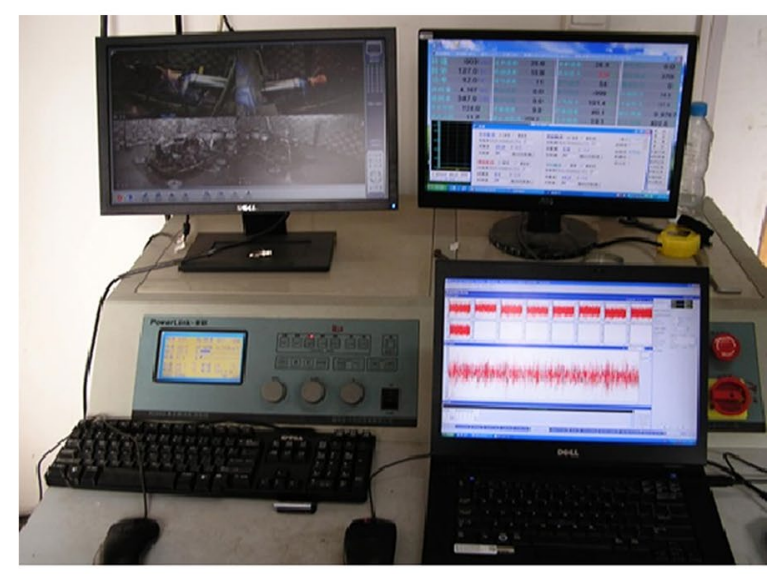

(b) Dynamometer and test system

Figure 2 Configuration of the experimental test rig

Table 1 Diesel engine valve clearance fault conditions

\begin{tabular}{lll}
\hline Condition & $\begin{array}{l}\text { Intake valve } \\
\text { clearance }(\mathbf{m m})\end{array}$ & $\begin{array}{l}\text { Exhaust valve } \\
\text { clearance }(\mathbf{m m})\end{array}$ \\
\hline Fault 1 & 0.20 & 0.40 \\
Fault 2 & 0.25 & 0.45 \\
Fault 3 & 0.30 & 0.50 \\
Fault 4 & 0.35 & 0.55 \\
Fault 5 & 0.40 & 0.60 \\
\hline
\end{tabular}

response will change. Changing the gas exchange process will influence the combustion process. The cylinder pressure will change accordingly and the vibration response of cylinder head, cylinder block, piston and other engine parts may also be different. These changes impact the whole integrated engine system, changing the nonlinear character of the engine vibration response.

Quadratic phase coupling (QPC) is the additive relationship between different components' frequencies and phases. If the harmonic components are irrelative, the third-degree cumulant is equal to 0 . If QPC exists in harmonic components, the third-degree cumulant is not equal to 0 . The bispectrum image has a peak value when analyzing the vibration signal with bispectrum of third-order cumulant. This indicated that QPC exists in vibration signals. At least in theory, the bispectrum can perfectly suppress the Gaussian components, and explain the distribution and strength of non-Gaussian components in the dual frequency domain, and also the QPC information. Fractal dimension shows the complexity of images, reflecting the quantification of the character of images. By using the fractal dimension, a quantificational description can be made, allowing evaluation of the engine condition and fault diagnosis. The Signal-flow diagram demonstration of the engine culpability diagnosis scheme is shown in Figure 4.

\subsection{Bispectrum Analysis}

Figure 5 shows the vibration signals in Locations 3 of the third cylinder under the five different valve clearance conditions. From the diagram, we can see that the surface of the cylinder trembling signal is composed of an array of shock response signals in a certain sequence, indicating the cycle stationary signal under the steady speed. Each signal under different conditions varied slightly in the time domain waveform because in the different states of valve mechanism, the mechanical stimulation including the impact of the valve stem, the rocker arm and valve seating are different. At the same time the difference of the intake and exhaust cause a change in the combustion status, leading to a change in the combustion excitation. Furthermore, for the diesel engine work process, there is fluctuation in the working cycle and the adjacent 


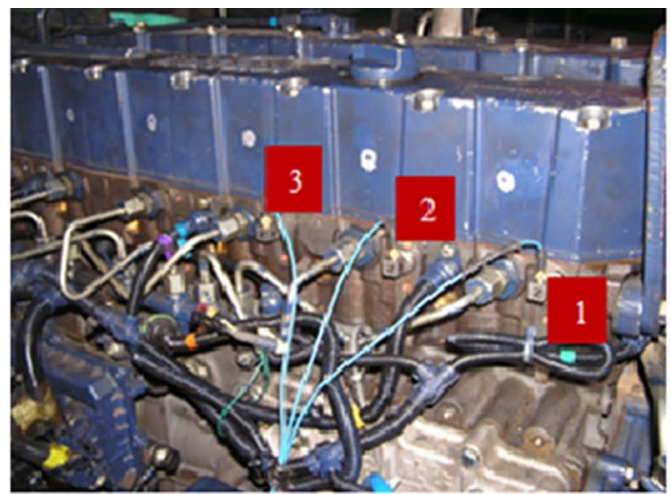

(a) 1-3 sensors

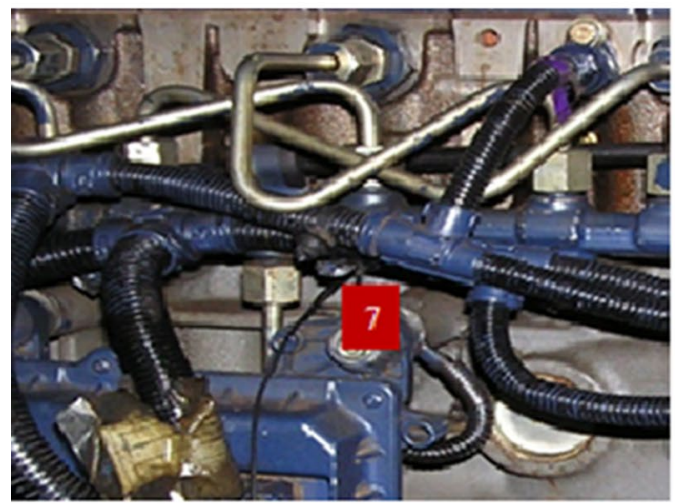

(c) 7 sensor

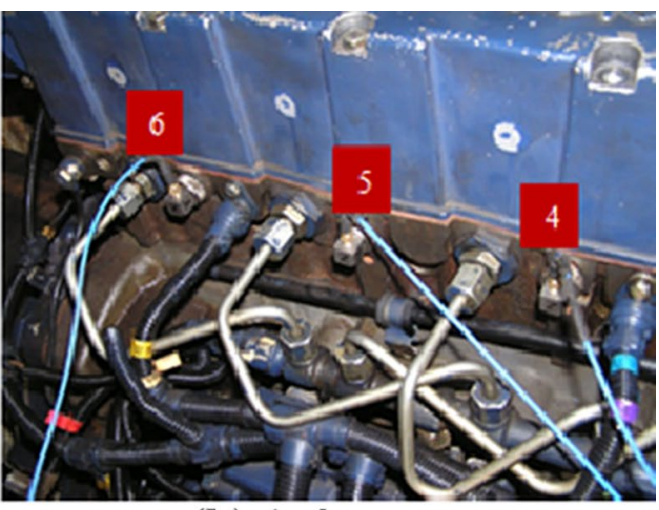

(b) 4-6 sensors

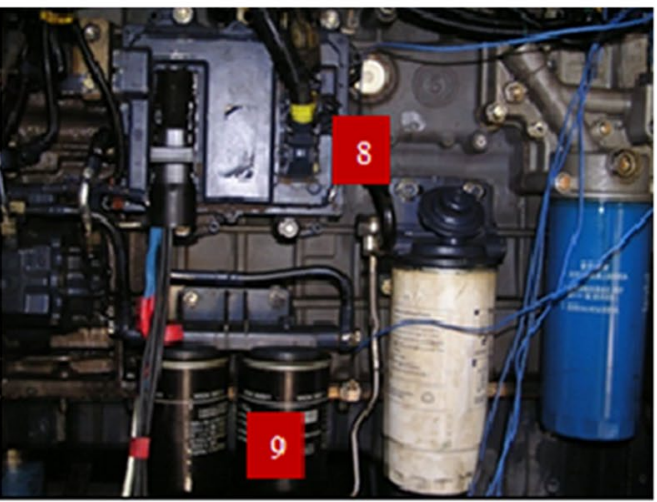

(d) 8-9 sensors

Figure 3 Locations of accelerative vibration sensors

cycle. All of these contribute to the vibration signal, and the cylinder head vibration response in the time domain waveform experiences fluctuation.

The nonlinear characteristics of the system have a significant impact on the output signal. Determining frequency relationship between the system and its output signal can identify the nonlinear characteristics. Because the signal bispectrum is highly sensitive to its non-Gaussian components, when these non-Gaussian characteristics of the system signal changes, the bispectrum features will change too. Here, the parametric bispectrum estimation was used to analyze the cylinder head vibration signal. The size of information is $N=25,600$, the request of every subsection perception tests $M=1024$, the superpose level of every subsection information is half, and the quantity of subsection is $K=2 N / M-1=49$. Next, figure the bispectrum of the vibration motion in Location 3 of the third barrel under the five conditions. The amplitudes and shapes are appeared in Figure 6. Normalizing frequency $f 1$ and $f 2$ based on the maximum 


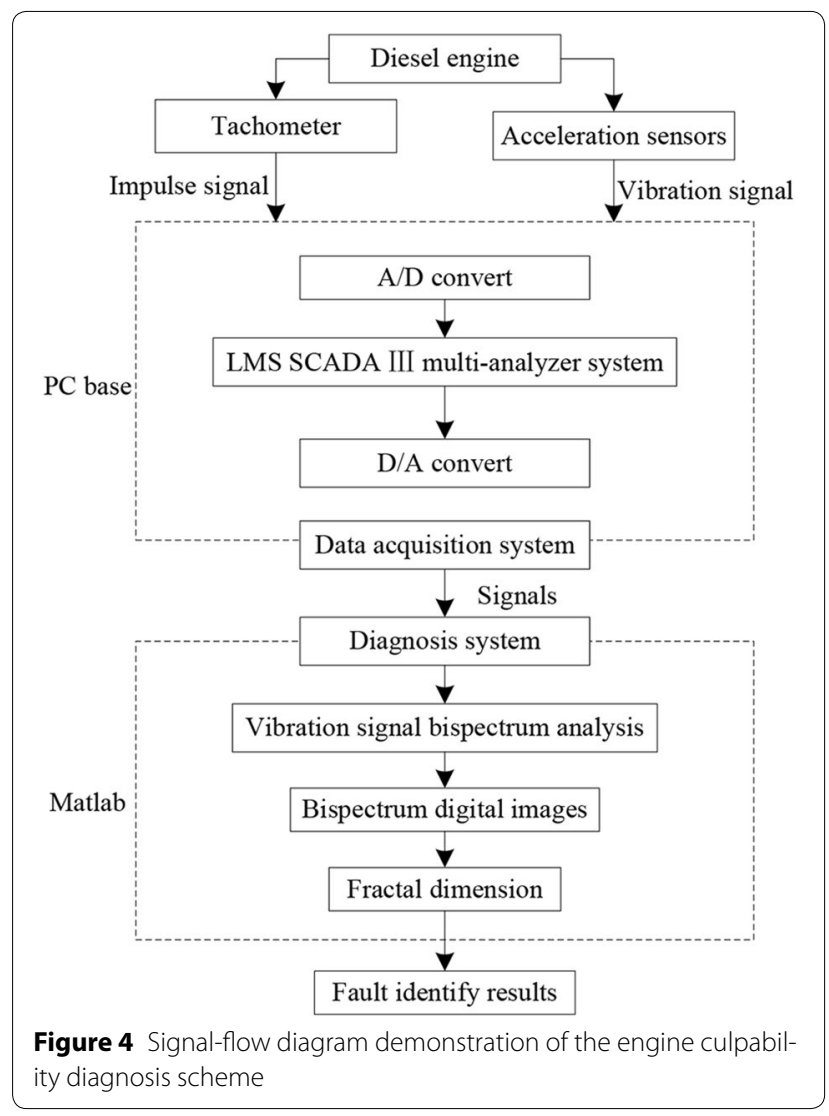

frequency, the frequency $f 1$ and $f 2(0-0.5 \mathrm{~Hz})$ correspond to $0-12,800 \mathrm{~Hz}$, and the frequencies below are normalized values.

In Figure 6, the cylinder head trembling signal for different states of the spectrum and amplitude are not zero, indicating that the cylinder trembling signal is a nonlinear and non-Gaussian signal. The bispectrum under the same condition is stable, but under various working conditions, the bispectrum shows obvious separability. Compared with the normal state (Fault 3), the bispectrum peak and phase distribution in other fault status is different, and the bispectrum distribution of the normal state is more complex. Using the normal state as a benchmark, from fault 1 to fault 3, the phase distribution of the bispectrum became gradually complicated, and from fault 3 to fault 5 , the phase distribution of state varied conversely. From the bispectrum amplitudes figure, we can see that the spectral peak amplitude of fault 3 (normal state) is lower than the other four kinds of fault states, because the valve clearance led to changes in the cylinder head trembling of nonlinear features. The degree of nonlinearity of the cylinder head system changes constantly with the deterioration of the condition (due to wear and deformation of the working parts).

\subsection{Fractal Dimension}

To determine the effect of valve clearance fluctuations in the fractal size of the vibration signal bispectrum, the bispectrum image was converted into a digital image and equivalent images were treated to evaluate the fractal size. Along with the clearance increase (before fault 3 ), the peak frequency became larger and the frequency components became complex and then (after fault 3) became simple (Figure 7a). The contours of the corresponding images increased and then decreased near fault 3 (Figure $7 b$ ).

For the five different valve clearance diesel engine conditions, the bispectrum contour maps of fractal dimension show obvious differences. The fault 3 fractal dimension is the largest. From state 1 to state 3 (normal condition), the fractal dimension increased as the valve clearance increased. This is likely because the valve opens earlier and closes loosely due to heat expansion under state 1 , causing cylinder leakage and a drop in combustion pressure. Under this condition, quadratic phase coupling of vibration signal stimulated by cylinder pressure (including both low frequency excitation and high frequency oscillation) decreases. Compared to the normal condition (state 3 ), the plot of the bispectrum was more simple with less side-band and center band components. The fractal dimension is also smaller. Under state 5 with too big valve clearance, the valve timing changed with both the intake and exhaust valve open, and the valve lift was also reduced. Therefore the gas exchange process is compressed, resulting in insufficient intake, partial exhaust, and incomplete combustion. The cylinder pressure also drops under incomplete combustion, causing a reduction in quadratic phase coupling and a simpler bispectrum of vibration signal. 

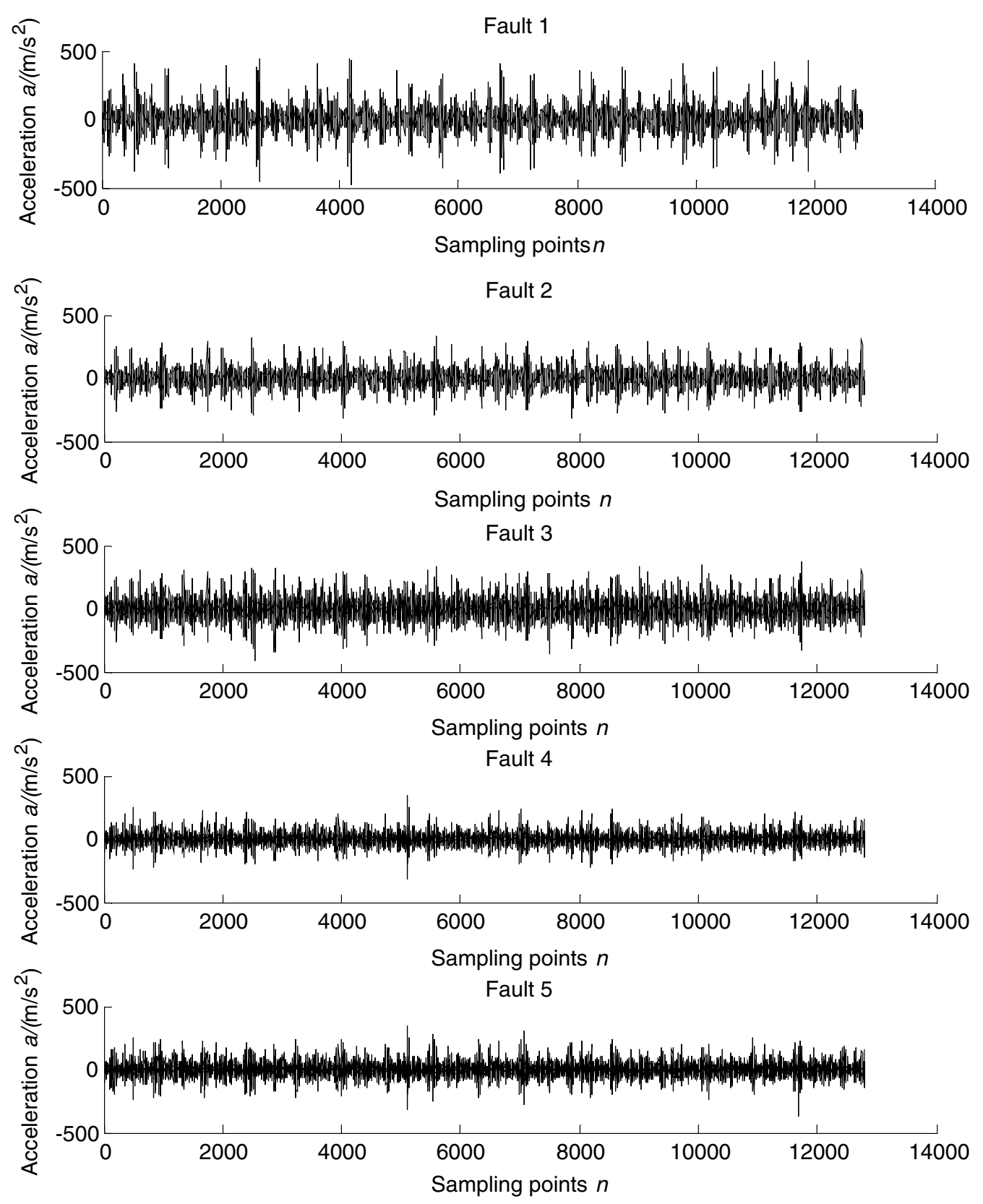

Figure 5 Time waveforms of vibration signals 

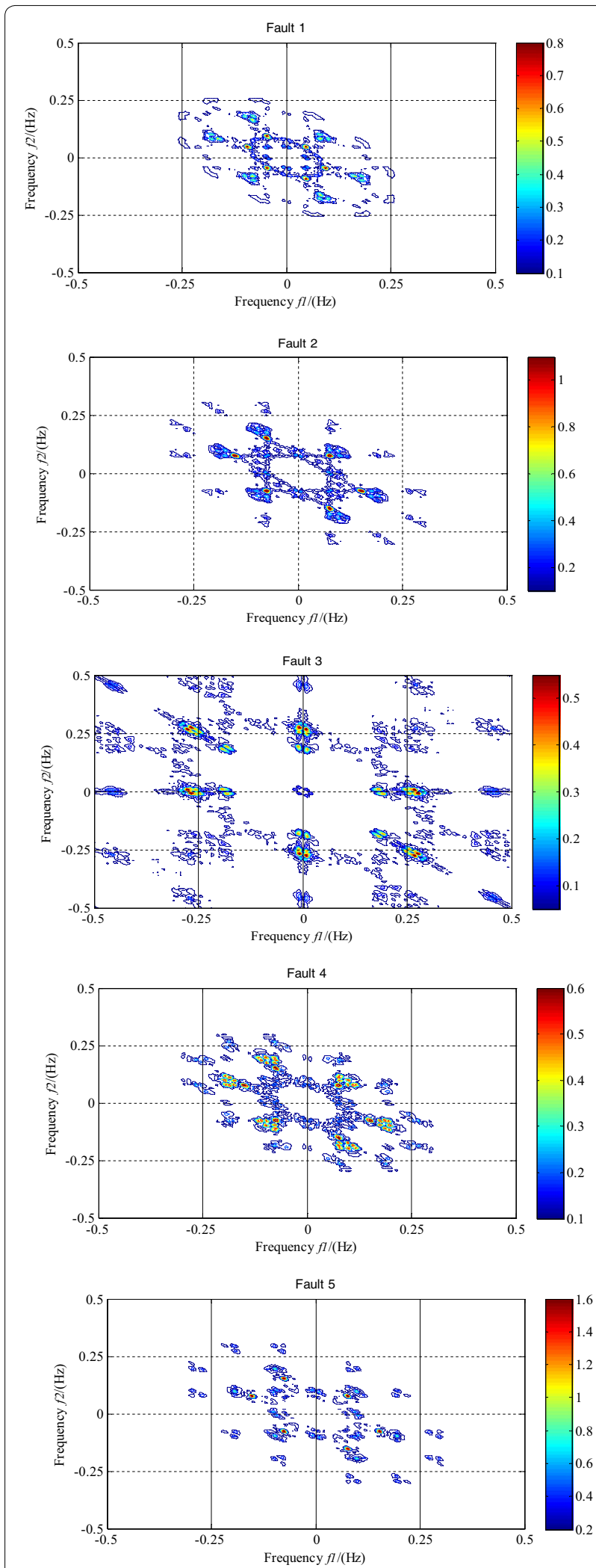

Figure 6 Vibration bispectrum at Location 3 of the third cylinder for the five conditions
Compared to the vibration signal acquired at $1600 \mathrm{r} /$ min with $40 \%$ load at 9 locations, the signal at location 3 was preferable. In order to accurately acquire the fault feature, 10 sets of data were intercepted respectively from the different time domain vibration signal under each fault condition. Each fragment contained 26.67 working cycle and lasted $1 \mathrm{~s}: 2-3 \mathrm{~s}, 5-6 \mathrm{~s}, 7-8 \mathrm{~s}, 10-11 \mathrm{~s}$, $13-14$ s, $16-17$ s, $19-20$ s, $22-23$ s, $24-25$ s, $27-28$ s. Next, the corresponding signals were processed to calculate the fractal dimensions. The results are shown in Figure 8.

Figure 8 shows the bispectrum fractal dimensions of 10 sets of vibration signal for each fault condition. The bispectrum fractal dimensions of the vibration signal under different valve clearance conditions showed obvious differences. Under the same condition, the fractal dimensions were similar and almost in the specific range. The small difference of the bispectrum fractal dimension under the same conditions were caused by changes in the non-Gaussian and nonlinear vibration signal with the fluctuations of the diesel engine working cycle and cycle-to-cycle working conditions. This underlying problem cannot be thoroughly solved, even if signals from more cycles are selected to reduce the error. Fractal dimensions under different fault states are in different ranges. When the diagnosis of conditions is consistent with the certain condition, the fractal dimension will be within the range of the specific conditions, allowing fault diagnosis.

\section{Conclusions}

(1) The vibration signal of engine is a non-linear, nonGaussian and cyclostationary signal. By using the bispectrum image and fractal dimension, characteristics that can reflect fault condition can be extracted from the vibration signals.

(2) The bispectrum distribution of the normal state is more complex. Using the normal state as a benchmark, from fault 1 to fault 3 , the phase distribution of the bispectrum became gradually complicated, and from fault 3 to fault 5 , the phase distribution of state varied conversely.

(3) Under different states, the bispectrums of vibration signal have different fractal dimensions, and these dimensions are in different ranges. Thus the fractal dimension can describe the working condition of engines. Additionally, the fractal dimension is consistent with the non-Gaussian characteristic of the signal. Therefore, the fractal dimension can serve as an index of the Gaussianity.

(4) The fractal dimension of the bispectrum increases at first then decreases as the valve clearance increased. Considering the discrepancy of engines, 


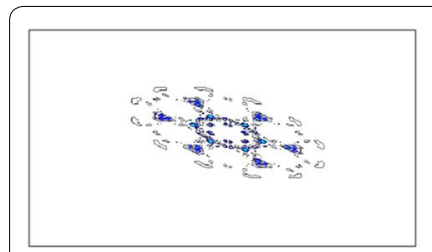

Fault 1

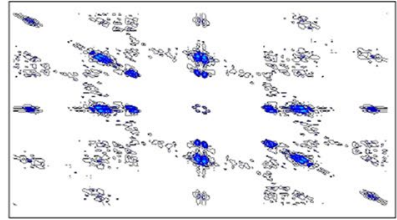

Fault 3

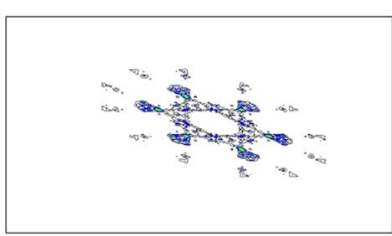

Fault 2

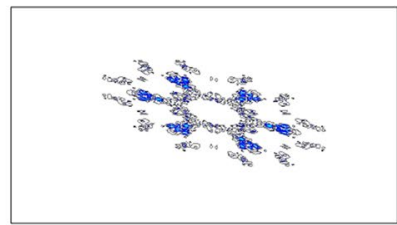

Fault 4

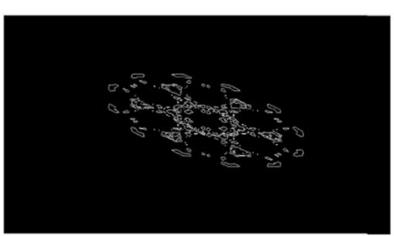

Fault $1(\mathrm{FD}=1.4623)$

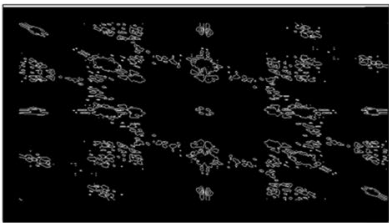

Fault $3 \mathrm{FD}=1.6898$

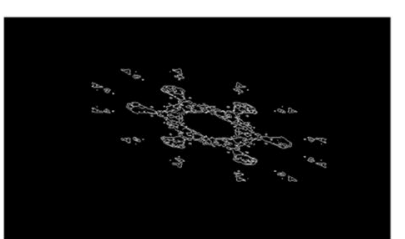

Fault $2 \mathrm{FD}=1.4802$

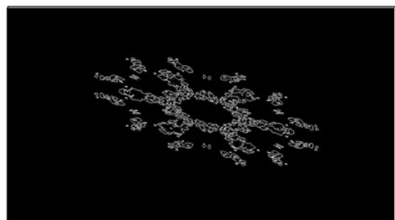

Fault $4 \mathrm{FD}=1.5382$

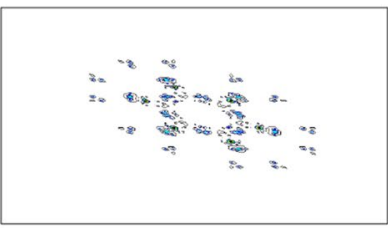

Fault 5

(a) Plots of bispectrum

Figure 7 Bispectrum evolution of five engine valve clearance conditions

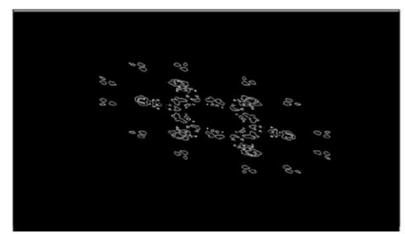

Fault $5 \mathrm{FD}=1.3788$

(b) Processed images and its FD

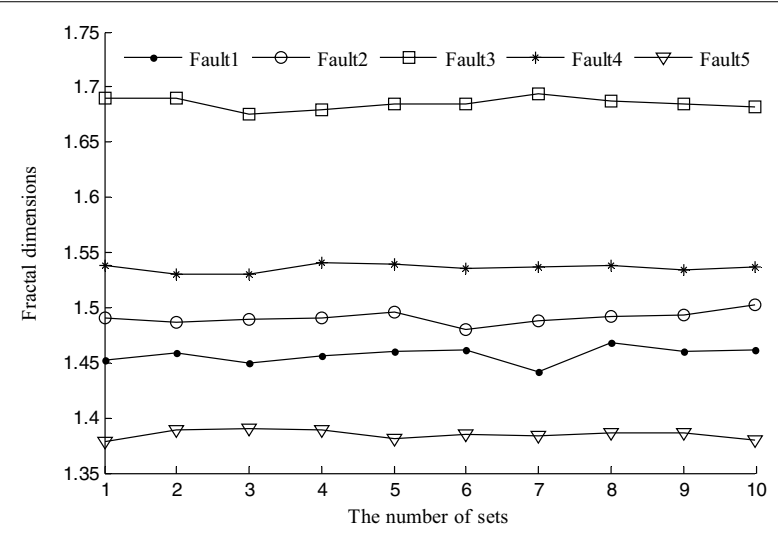

Figure 8 Fractal dimensions of 10 sets of data under each fault condition

the fractal dimension under the same conditions can be different. Building a database could help to solve this problem.

\section{Authors' Contributions}

F-RB was in charge of the whole trial; JZ wrote the manuscript; C-WL, X-BB, $X Y$ assisted with sampling and laboratory analyses. All authors have read and approved the final manuscript.

\section{Authors' Information}

Jian Zhang, born in 1983, is currently a PhD candidate at State Key Laboratory of Engine, Tianjin University, China. He received his master degree from Kunming University of Science and Technology, China, in 2012. His research interests include engine noise and vibration signal processing. Tel: +86-17720531162; E-mail: neil1101@tju,edu.cn.

Chang-Wen Liu, born in 1963, is currently a professor at State Key Laboratory of Engine, Tianjin University, China. His research interest is engine's electronic control technology. Tel: +86-13602057489; E-mail: liuchangwen@ tju.edu.cn.

Feng-Rong Bi, born in 1965, is currently a professor at State Key Laboratory of Engine, Tianjin University, China. He received his PhD degree from Tianjin University, China, in 2003. His research interests include engine noise and vibration control, automobile dynamics, etc. Tel: +86-13802167153; E-mail: fr_bi@ tju.edu.cn.

Xiao-Bo Bi, born in 1978, is currently a PhD candidate at State Key Laboratory of Engine, Tianjin University, China. He received his master degree from Hebei University of Technology, China, in 2014. His research interests include diesel engine fault diagnosis. Tel: +86-13820411609; E-mail: $1014201001 @$ tju. edu.cn.

Xiao Yang, born in 1993, is currently a master candidate at State Key Laboratory of Engines, Tianjin University, China. He received his bachelor degree from Tianjin University, China, in 2015. His research interests include engine noise control. Tel: +86-18920965767; E-mail: yangxiao@tju.edu.cn.

\section{Competing Interests}

The authors declare that they have no competing interests.

\section{Ethics Approval and Consent to Participate}

Not applicable. 


\section{Funding}

Supported by National Science and Technology Support Program of China (Grant No. 2015BAF07B04).

\section{Publisher's Note}

Springer Nature remains neutral with regard to jurisdictional claims in published maps and institutional affiliations.

Received: 2 February 2016 Accepted: 13 April 2018

Published online: 08 May 2018

\section{References}

[1] G Gelle, M Colas, C Serviere. Blind source separation: a tool for rotating machine monitoring by vibration analysis. Journal of Sound \& Vibration, 2001, 248(5): 865-885.

[2] K Shibata, A T Takahashi. Fault diagnosis of rotating machinery through visualization of sound signals. Mechanical Systems and Signal Processing 2000, 14(14): 229-241.

[3] Z M Geng, J Chen, J B Hull. Analysis of engine vibration and design of an applicable diagnosis approach. International Journal of Mechanical Sciences, 2013, 45(8): 1391-1410.

[4] W Q Wang, I Fathy, M F Golnaraghi. Assessment of gear damage monitoring techniques using vibration measurements. Mechanical Systems and Signal Processing, 2001, 15(5): 905-922.

[5] H Z Gao, L Liang, X G Chen, et al. Feature extraction and recognition for rolling element bearing fault utilizing short-time Fourier transform and non-negative matrix factorization. Chinese Journal of Mechanical Engineering, 2015, 28(1): 96-105

[6] J P Shao, H J Jia. Feature extraction of vibration signals based on wavelet packet transform. Chinese Journal of Mechanical Engineering, 2004, 17(1): 25-27.

[7] Y G Lei, Z J He, Y Y Zi, et al. New clustering algorithm-based fault diagnosis using compensation distance evaluation technique. Mechanical System and Signal Processing, 2008, 22(2): 419-435.

[8] X G Chen, L Liang, G H Xu, et al. Feature extraction of kernel regress reconstruction for fault diagnosis based on self-organizing manifold learning. Chinese Journal of Mechanical Engineering, 2013, 26(5): 1041-1049.

[9] J D Wu, J C Chen. Continuous wavelet transform technique for fault signal diagnosis of internal combustion engines. Ndt\&E International, 2006, 39(4): 304-311

[10] WT Peter, W X Yang, HY Tam. Machine fault diagnosis through an effective exact wavelet analysis. Journal of Vibration \& Acoustics, 2004, 227(4-5): 1005-1024.

[11] XWang, CW Liu, F R Bi, et al. Fault diagnosis of diesel engine based on adaptive wavelet packets and EEMD-fractal dimension. Mechanical Systems and Signal Processing, 2013, 41(1): 581-597.

[12] B Liang, S D Iwnicki, Y Zhao. Application of power spectrum, higher order spectrum and neural network analyses for induction motor fault diagnosis. Mechanical Systems and Signal Processing, 2013, 39(1-2): 342-360.

[13] M Li, J H Yang, X J Wang. Fault feature extraction of rolling bearing based on an improved cyclical spectrum density method. Chinese Journal of Mechanical Engineering, 2015, 28(6): 1240-1247.

[14] M J Zhang, J Tang, X M Zhang, et al. Intelligent diagnosis of short hydraulic signal based on improved EEMD and SVM with few low-dimensional training samples. Chinese Journal of Mechanical Engineering, 2016, 29(2): 396-405.

[15] K J Shi, S L Liu, C Jiang, et al. Rolling bearing feature frequency extraction using extreme average envelope decomposition. Chinese Journal of Mechanical Engineering, 2016, 29(5): 1029-1036.

[16] F S Gu, A Naid, N Q Hu, et al. Electrical motor current signal analysis using modified bispectrum for fault diagnosis of reciprocating compressors. Condition Monitoring \& Diagnostic Engineering Management, San Sebastian, Spain, June 9-11, 2009.

[17] F A Gu, Y Shao, N Q Hu, et al. Electrical motor current signal analysis using modified bispectrum for fault diagnosis of downstream mechanical equipment. Mechanical Systems and Signal Processing, 2011, 25(1): 360-372.

[18] G J Shen, S Mclaughlin, Y C Xu, et al. Theoretical and experimental analysis of bispectrum of vibration signals for fault diagnosis of gears. Mechanical Systems and Signal Processing, 2014, 43 (s1-2): 76-89.

[19] F Z Feng, A W Si, HX Zhang. Research on fault diagnosis of diesel engine based on bispectrum analysis and genetic neural network. Procedia Engineering, 2011, 15(1): 2454-2458.

[20] H M Zhao, CY Xia, Y K Xiao, et al. Bispectrum analysis for vibration data of crankshaft cearing in diesel engine. Journal of Vibration, Measurement \& Diagnosis, 2009, 29(1): 14-18. (in Chinese).

[21] T Li, S Chen, Q Tang, et al. Fault Diagnosis for valve train of diesel engine based on bispectrum estimation via non-gaussian AR model. Chinese Internal Combustion Engine Engineering, 2010, 31(1): 82-87. (in Chinese)

[22] Y Liu, L Y Chen, H M Wang, et al. An improved differential box-counting method to estimate fractal dimensions of gray-level images. Journal of Visual Communication and Image Representation, 2014, 25(5): 1102-1111.

[23] J Guo, X Guo, P F Luo. A new method for antomatic target recognition. Proceeding of the IEEE Nation Aerospace and Electronics Conference NAECON, Dayton, $\mathrm{OH}$, Jul 14-18, 1997, 2: 1019-1024.

[24] D R Brillinger. An introduction to polyspectra. Annals of Mathematical Statistics, 1965, 36(5): 1351-1374.

[25] D R Brillinger. Some basic aspects and uses of higher order spectra. Signal Process, 1994, 36(3) 239-249.

[26] N Otsu. A threshold selection method from gray-level histograms. IEEE Transactions on Systems Man \& Cybernetics, 1979, 9(1): 62-66.

[27] Y Y Duan, L Wang, H Z Chen, Digital image analysis and fractal-based kinetic modelling for fungal biomass determination in solid-state fermentation. Biochemical Engineering Journal, 2012, 67(1): 60-67.

[28] L Zhao, Z D Zhou, Y Yang, et al. Feature extraction of rolling bearing fault based on ensemble empirical mode decomposition and correlation dimension. International Manufacturing Science and Engineering Conference, Detroit, Michigan, USA, June 9-13, 2014.

[29] J Theiler. Estimating fractal dimension. Journal of the Optical Society of America A, 1990, 7(6): 1055-1073.

[30] D P Donnelly, L Boddy, J R Leake. Development, persistence and regeneration of foraging ectomycorrhizal mycelial systems in soil microcosms. Mycorrhiza, 2004, 14(1): 37-45.

\section{Submit your manuscript to a SpringerOpen ${ }^{\oplus}$ journal and benefit from:}

- Convenient online submission

- Rigorous peer review

- Open access: articles freely available online

- High visibility within the field

Retaining the copyright to your article

Submit your next manuscript at springeropen.com 